\title{
Human Mutation
}

WILEY

\section{Hereditary fructose intolerance: functional study of two novel ALDOB natural variants, and characterization of a partial gene deletion}

\begin{tabular}{|r|l|}
\hline Journal: & Human Mutation \\
\hline Manuscript ID: & humu-2010-0109.R1 \\
\hline Wiley - Manuscript type: & Research Article \\
\hline Duthor: & 29-Jul-2010 \\
\hline Complete List of Authors: & $\begin{array}{l}\text { Esposito, Gabriella; CEINGE - Biotecnologie Avanzate; Universita' } \\
\text { degli Studi di Napoli "Federico II", Dipartimento di Biochimica e } \\
\text { Biotecnologie Mediche; IRCCS-Fondazione SDN } \\
\text { Imperato, Maria Rosaria; CEINGE - Biotecnologie Avanzate } \\
\text { Ieno, Luigi; CEINGE - Biotecnologie Avanzate; Universita' degli } \\
\text { Studi di Napoli "Federico II", Dipartimento di Biochimica e } \\
\text { Biotecnologie Mediche } \\
\text { Sorvillo, Rosa; CEINGE - Biotecnologie Avanzate; Universita' degli } \\
\text { Studi di Napoli "Federico II", Dipartimento di Biochimica e } \\
\text { Biotecnologie Mediche } \\
\text { Benigno, Vincenzo; Ospedale S. Giovanni di Dio, Unità Operativa di } \\
\text { Pediatria } \\
\text { Parenti, Giancarlo; Universita' degli Studi di Napoli "Federico II", } \\
\text { Dipartimento di Pediatria } \\
\text { Parini, Rossella; Ospedale S. Gerardo, Clinica Pediatrica } \\
\text { Vitagliano, Luigi; CNR, Istituto di Biostrutture e Bioimmagine } \\
\text { Zagari, Adriana; Università degli studi di Napoli, Dipartimento di } \\
\text { Scienze Biologiche \& CNISM } \\
\text { Salvatore, Francesco; CEINGE - Biotecnologie Avanzate; IRCCS- } \\
\text { Fondazione SDN }\end{array}$ \\
\hline Key Words: & $\begin{array}{l}\text { missense mutations, ALDOB kinetic analysis, ALDOB molecular } \\
\text { modeling, HFI molecular diagnosis, HFI molecular epidemiology, } \\
\text { intragenic deletion }\end{array}$ \\
\hline \hline
\end{tabular}

\section{SCHOLARONE Manuscripts}




\title{
Hereditary fructose intolerance: functional study of two novel $A L D O B$
}

\section{natural variants, and characterization of a partial gene deletion}

\author{
${ }^{*+\dagger}$ Gabriella Esposito, ${ }^{*}$ Maria Rosaria Imperato, ${ }^{* \dagger}$ Luigi Ieno, ${ }^{* \dagger}$ Rosa Sorvillo, ${ }^{\S}$ Vincenzo \\ Benigno, ${ }^{q /}$ Giancarlo Parenti, ${ }^{\S \S}$ Rossella Parini, ${ }^{\varpi}$ Luigi Vitagliano, ${ }^{* \#}$ Adriana Zagari, \\ ${ }^{*}$ Francesco Salvatore. \\ ${ }^{*}$ CEINGE-Biotecnologie Avanzate S.C.a r.l., Napoli, Italy; ${ }^{\dagger}$ Dipartimento di Biochimica e Biotecnologie Mediche, \\ Università degli Studi di Napoli “Federico II”, Napoli, Italy; "IRCCS-Fondazione SDN, via E. Gianturco 113, 80143 \\ Napoli, Italy; ${ }^{\S}$ Unità Operativa di Pediatria, Ospedale S. Giovanni di Dio, Agrigento, Italy, ${ }^{\mathbb{I}}$ Dipartimento di Pediatria,

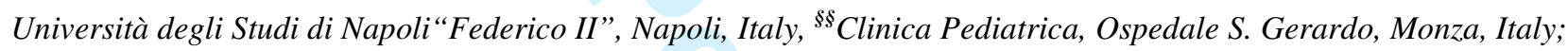 \\ ${ }^{\varpi}$ Istituto di Biostrutture e Bioimmagine, CNR, Napoli, Italy; ${ }^{\#}$ Dipartimento delle Scienze Biologiche -Sezione Biostrutture \\ e CNISM, Università degli Studi di Napoli “Federico II”, Napoli, Italy.
}

\begin{abstract}
Hereditary fructose intolerance (HFI) is an autosomal recessive metabolic disease caused by impaired functioning of human liver aldolase (ALDOB). At least 54 subtle/point mutations and only two large intragenic deletions have been found in the $A L D O B$ gene. Here we report two novel ALDOB variants (p.R46W and p.Y343H) and an intragenic deletion that we found in patients with suspected HFI. The residual catalytic activity of the recombinant p.R46W and p.Y343H variants towards F1P was particularly altered. We also characterized a large intragenic deletion that we found in six unrelated patients. This is the first report of six unrelated patients sharing the same $A L D O B$ deletion, thus indicating a founder effect for this allele in our geographic area. Because this deletion involves $A L D O B$ exon 5, it can mimic worldwide common pathogenic genotypes, i.e., homozygous p.A150P and p.A175D. Finally, the identification of only one $A L D O B$ mutation in symptomatic
\end{abstract}


patients suggests that HFI symptoms can, albeit rarely, appear also in heterozygotes. Therefore, an excessive and continuous fructose dietary intake may have deleterious effects even in apparently asymptomatic HFI carriers.

\begin{abstract}
Key words: missense mutations, ALDOB kinetic analysis, ALDOB molecular modeling, intragenic deletion, HFI molecular diagnosis, HFI molecular epidemiology.
\end{abstract}

\title{
Introduction
}

It is now becoming increasingly recognized that even in healthy individuals excessive fructose intake may cause a series of adverse effects that, in the long term, are associated to the "metabolic syndrome" [Miller and Adeli, 2008]. Obviously, the negative effects of chronic fructose loading may be particularly pronounced in carriers of inborn errors of fructose metabolism [Steinmann et al., 2001]. A case in point is hereditary fructose intolerance (HFI; MIM\# 229600), an autosomal recessive severe disease of infants and young children that is due to a deficiency of the liver-specific B isoform of the fructose-1,6-bisphosphate aldolase (ALDOB; MIM *612724).

Aldolases (EC 4.2.1.13) are homotetrameric isoenzymes that catalyze the reversible aldol cleavage of fructose 1,6-bisphosphate (FBP) and fructose 1-phosphate (F1P). There are three tissue-specific mammalian aldolase isoenzymes: aldolase A (ALDOA; MIM *103850) in muscle, ALDOB in liver, small intestine and kidney, and aldolase C (ALDOC; MIM *103870) in brain [Salvatore et al., 1986]. In liver, fructokinase (EC 2.7.1.3) rapidly phosphorylates dietary fructose to F1P that is specifically metabolized by ALDOB in the gluconeogenic/glycolytic pathway. In individuals affected by HFI, ingestion of fructose and sucrose results in accumulation of F1P and produces acute and chronic symptoms. Symptoms such as abdominal pains, vomiting, and serious metabolic disturbances, 
including severe hypoglycemia, appear after starting an HFI infant on sweetened milk formulae, solid foods, fruits and vegetables. Additional symptoms are renal tubular acidosis with hyperuricemia and hypertransaminasemia. Prolonged fructose intake leads to poor feeding, growth failure, jaundice, hepatomegaly, hemorrhage and progressive liver damage, which may be fatal in some cases [Steinmann et al., 2001]. Disease symptoms usually disappear with a totally fructose-free diet. After the difficult initial period of weaning, HFI infants usually develop a self-protective aversion to foods that cause distress. HFI can thus remain undiagnosed for a long time in patients who adhere to a "self-imposed" low fructose diet. However, even in these cases, HFI remains a problem due to recurrent inadvertent fructose ingestion. Deaths have been documented in undiagnosed HFI individuals who have been challenged unintentionally with fructose (or cognate sugars), for example, in parenteral feeding [Ali et al., 1998].

The incidence rate of HFI varies among countries and ethnic groups. It is $1: 22,000$ in the UK [James et al., 1996], 1:26,100 in Central Europe [Santer et al., 2005] and 1:31,000 in Poland [Gruchota et al., 2006]. However, numerous reports of self-diagnosis in adulthood, death of undiagnosed subjects, and homozygous-heterozygous marriages, all indicate that the HFI incidence rate could be higher. Moreover, detected cases of HFI are increasing because of increased sugar consumption in industrialized countries [Cox, 2002].

Hereditary fructose intolerance is conventionally diagnosed by evaluating clinical symptoms upon fructose challenge or by measuring aldolase activity in liver or intestine biopsy samples [Steinmann and Gitzelmann, 1981]. Both methods are relatively invasive and represent considerable risk, especially in a newborn. Consequently, it is preferable to verify the clinical suspicion of HFI by the non-invasive molecular analysis of the $A L D O B$ gene (HGNC ID \#417).

The human $A L D O B$ gene, located on chromosome 9q22.3, is 14,500-bp long, contains nine 
exons, the first of which is untranslated and represents the promoter region. Exons 2-9 encode the type B monomer, a 364-amino acid long polypeptide; four identical monomers assemble to form the mature, tetrameric enzyme. The mutations identified to date in the $A L D O B$ gene of HFI patients consist mainly of subtle/point mutations (missense, nonsense, splicing defects and frameshift mutations) [Stenson et al., 2003; Sanchez-Gutierrez et al., 2002; Davit-Spraul et al., 2008; the Human Gene Mutation Database, http://www.hgmd.org] and only two large intragenic deletions [Cross and Cox, 1990]. Two missense mutations in exon 5, c.448G >C (p.A150P) and c.524C >A (p.A175D), account for about 70-80\% of all HFI alleles worldwide [Steinmann et al., 2001; Esposito et al., 2004a; Coffee et al., 2009]. The other mutations are spread throughout the ALDOB gene, and their frequency differs among ethnic groups [Steinmann et al., 2001]: some have become widespread as a result of increasing inter-marriage and genetic admixture, whereas others are private mutations [Ali et al., 1998].

Here we report three novel $A L D O B$ mutations that we found in putative HFI patients, i.e. two nucleotide changes that lead to the missense variants p.R46W and p.Y343H, and a large intragenic deletion.

\section{Material and Methods}

Patients We studied three children (AB, CL, and SR) affected by clinical symptoms suggestive of HFI from three unrelated Italian families. We also studied the asymptomatic daughter (MG) of a patient who was previously diagnosed as homozygous for a frequent HFI mutation - no history was available for this family because the mother was adopted. Patient $A B$ at the age of 8 months was hospitalized for a series of fever episodes associated with severe hypertransaminasemia and hepatomegaly. During hospitalization, in line with the liver malfunctioning, she also showed amino 
acid urea, and high bilirubin, LDH, ferritin, PT and PTT, which alerted the physician to the possibility of HFI. She died one month later, from unknown causes, ten days after HFI was suspected. CL had mild hypoglycemia and ketosis after ingestion of fructose, and a marked aversion to sweets and fruit. SR was undiagnosed until she was 10 years old; she had growth failure and mild mental retardation probably due to recurrent fructose ingestion. At the age of 3 months, her younger brother had episodes of severe hypoglycemia and hypertransaminasemia after ingestion of fructose. Subsequently, he developed a marked aversion to sweets and fruit; this led to the suspicion of HFI, which was confirmed by a fructose challenge. No data of fructose challenge or aldolase activity on liver biopsy were available for the other patients. Finally, we re-evaluated for deletions in $A L D O B$ four additional patients who were previously diagnosed as homozygous for c.448G $>$ C (p.A150P) or c.524C >A (p.A175D) in the absence of parents to check the Mendelian autosomal recessive transmission. Adherence to appropriate dietary restrictions resulted in complete regression of symptoms, except mental retardation, in all symptomatic patients. All heterozygous relatives were asymptomatic. Genetic investigations were performed after written informed consent of patients and/or parents. This study was performed according to the guidelines for genetic tests approved by the Ministero della Salute, Rome, Italy.

Mutation analysis Genomic DNA was extracted from blood samples with the Nucleon BACC2 kit (GE® Health Care Europe-Amersham, Chalfont St. Giles, U.K). Mutation numbering is based on gDNA (GenBank Accession \#NC_000009.11), cDNA (\#NM_000035.2) and protein (\#NP_000026.2) sequences; for cDNA numbering, +1 corresponds to the A of the ATG translation initiation codon, which is codon 1 . 
The amplification refractory mutation system (ARMS) was used to analyze the patients' DNA for the presence of seven known HFI mutations (Fig.1), which account for about $90 \%$ of the HFI Italian alleles [Esposito et al., 2004a]. The $A L D O B$ exons 2-9 with their flanking sequences, and the promoter region (the untranslated exon 1) were amplified as reported elsewhere [Santamaria et al., 1993], and sequenced with the Big Dye ${ }^{\mathrm{TM}}$ Terminator v.3.1 Sequencing kit and the ABI Prism 3730 DNA Analyzer (Applied Biosystems, Foster City, CA, USA). Novel point mutations detected by sequencing were confirmed by following the Mendelian transmission in parents and other members of the family. To verify that the novel point mutations are not neutral polymorphisms, we checked 300 alleles from 150 unaffected controls. The known $A L D O B$ intragenic deletions, namely g.75169165del and g.9912_10836del [Cross and Cox, 1990], were looked for with the primer pair 3F (5'CTTGCTTTCCACTGTGGTGA-3')/6R (5'-ATATGTTAAGTAACAGCTGTTAC-3'), and 5F (5'TAGAAGCCCCATGGATCAGG-3')/8R (5'-AAGAAAACAATGCTTCTCCG-3'), respectively. In both cases, the reaction mixture contained $0.2 \mu \mathrm{g}$ of genomic DNA, $1 \mathrm{U}$ Taq polymerase (Roche Diagnostics, Mannheim, Germany), $200 \mu \mathrm{M}$ of each dNTP, $50 \mathrm{mM} \mathrm{KCl,} 10 \mathrm{mM}$ Tris/HCl, pH 8.8, $2.5 \mathrm{mM} \mathrm{MgCl} 2,0.01 \%(\mathrm{w} / \mathrm{v})$ gelatin, in a final volume of $50 \mu \mathrm{l}$. After an initial denaturing step of 1 min at $94^{\circ} \mathrm{C}$, samples were amplified for 32 cycles at $94^{\circ} \mathrm{C}$ for $20 \mathrm{~s}, 61^{\circ} \mathrm{C}$ for $15 \mathrm{~s}$ and $72^{\circ} \mathrm{C}$ for 3.5 $\min$.

\section{Quantitative PCR (qPCR) and deletion breakpoint characterization}

Detection and fine mapping of the new $A L D O B$ deletion herein reported was performed by real time quantitative PCR (qPCR) reactions using the Power Sybr Green PCR Master kit (Applied Biosystems). We determined the difference in copy number between proband DNA and the DNA from two normal subjects, with duplicate samples for each experiment. We performed qPCR analysis 
of exons 1, 2, 6, 7, 9 using primers reported previously [Santamaria et al., 1993], and an additional primer pair $\left(I V S 1^{\mathrm{F}}\right.$ : 5'-TTCGCCTTCACCCACTGCTCA-3'; IVS1 ${ }^{\mathrm{R}} 5^{\prime}$ -

TCTAGGCACTGCAGCACTTTTG-3') to analyze the ALDOB intron 1. qPCRs were performed using the iCycler ${ }^{\mathrm{TM}}$ version 3.021 (Bio-Rad Laboratories S.r.l., Segrate, Italy) and the fluorescent signal intensity was recorded and analyzed by iCycler ${ }^{\mathrm{TM}}$ iQ Optical System software v3.0a (Bio-Rad Laboratories S.r.1.). A double copy $\beta$-globin gene fragment (HBB) was used as control. To calculate copy number we used the formula: copy number $=2 * 2^{-(\Delta \mathrm{Ctp}-\Delta \mathrm{Ctn})}$ where $\mathrm{Ct}$ was the threshold cycle defined as the mean cycle at which the fluorescence curve reached an arbitrary threshold; $\Delta \mathrm{Ct}$ was calculated as $\mathrm{Ct}$ of $A L D O B-\mathrm{Ct}$ of $H B B, \triangle \mathrm{Ctp}$ was the $\triangle \mathrm{Ct}$ of proband, and $\triangle \mathrm{Ctn}$ was the $\triangle \mathrm{Ct}$ of normal individuals. The deletion breakpoint region was amplified using $50 \mathrm{ng}$ of the proband's genomic DNA, 10 pmoles of primers IVS1.1F (5'-CCTAGAAATCATGGGCTATC-3') and 7.2R (5'-CAAACAGAAAGCTTGTGGCT-3') in a final volume of $50 \mu$ l containing $200 \mu \mathrm{M}$ of dNTPs, $2.5 \mathrm{mM}$ of $\mathrm{MgCl}_{2}, 2 \%$ DMSO, $1 \mathrm{U}$ Taq DNA polymerase (Roche Diagnostics) and the following cycling parameters: $95^{\circ} \mathrm{C}$ for $3.5 \mathrm{~min}, 30$ cycles at $95^{\circ} \mathrm{C}$ for $25 \mathrm{sec}, 58^{\circ} \mathrm{C}$ for $20 \mathrm{sec}$ and $72^{\circ} \mathrm{C}$ for 3 min; final extension: $72^{\circ} \mathrm{C}$ for $10 \mathrm{~min}$. The resulting PCR product (about $2.4 \mathrm{~kb}$ ), which spanned the deletion breakpoint, was sequenced. Consequently, we were able to set-up a duplex PCR to amplify the breakpoint junction together with an internal control, to rapidly identify subjects who were heterozygous for this deletion. Primers BPF (5'-CCAGCTAGATTAGGCAGAGC-3') in IVS1 and $B P R\left(5^{\prime}\right.$-TATGACTTGCCCAAGATCCC-3') in IVS6 give rise to a 258-bp fragment that contains the deletion junction. The internal amplification control was an $H B B$ amplicon of about $357 \mathrm{bp}$. SINE and LINE sequences in the $A L D O B$ were searched using the RepeatMasker web server (http://www.repeatmasker.org/cgi-bin/WEBRepeatMasker). 


\section{Site-directed mutagenesis, expression, purification and kinetic studies of the recombinant}

\section{ALDOB enzymes}

We used an N-terminal polyhistidine (His)-tagged human ALDOB expressing vector [Santamaria et al., 2000] as a template to generate the enzyme variants p.R46W and p.Y343H, according to the instructions in the QuickChange ${ }^{\mathrm{TM}}$ site-directed mutagenesis kit (Stratagene Inc., La Jolla, CA). Wild type and mutated recombinant plasmids were sequenced and used to transform the bacterial strain $E$. coli BL21(DE3) cells as described previously [Santamaria et al., 2000; Esposito et al., 2002]. Activity assays and kinetic studies on the purified His-tagged recombinant enzymes corresponding to the wild-type, p.R46W and p.Y343H ALDOB were performed at $30^{\circ} \mathrm{C}$, as previously described [Santamaria et al., 2000]. The thermal stability of the recombinant p.R46W, p.Y343H and normal ALDOB was monitored by measuring specific activity versus FBP and F1P at temperatures that
varied in the range of $20-50^{\circ} \mathrm{C}$ [Esposito et al., 2005]. The data were statistically analyzed using
Student t test. The level of significance was set at $\mathrm{P}<0.01$.

\section{Circular dichroism experiments}

The circular dichroism (CD) spectra of the recombinant wild-type ALDOB and of the two missense variants were recorded with a Jasco J-810 spectropolarimeter equipped with a Peltier temperature control system (Model PTC-423-S). Far-UV measurements (198-250 nm) were carried out using a $0.1 \mathrm{~cm}$ optical path length cell and a protein concentration of $0.15 \mathrm{mg}^{\circ} \mathrm{ml}^{-1}$. Denaturation curves were recorded by following the $\mathrm{CD}$ signal at $222 \mathrm{~nm}$.

\section{Molecular graphics analyses}

The mutation-induced structural alterations were studied by using three-dimensional structures of 


\begin{abstract}
ALDOB reported in the Protein Data Bank (PDB). In particular, the following ALDOB crystal structures were considered: (a) the ligand-free form (PDB code 1QO5) [Dalby et al., 2001], (b) the mutant p.A150P (PDB code 1XDL and 1XDM) [Malay et al., 2005], and (c) the intermediate state along the reaction pathway (PDB code 1FDJ). The analyses were conducted with the PROMOTIF [Hutchinson et al., 1996], O [Jones et al., 1991], and PROCHECK [Laskowski et al., 1993] programs. The energy of the 3D-models of ALBOB mutants has been minimized using the program GROMACS [Van Der Spoel et al., 2005]. As the 343 mutation site is exposed to the solvent, for the p.Y343H variant, His343 and the nearby His345 were considered uncharged.
\end{abstract}

\title{
Results and Discussion
}

Here, we report cases in which the molecular analysis of the whole $A L D O B$ gene, carried out to verify a clinical suspicion of HFI, revealed a novel large intragenic deletion (g.2840_9288del), and two single-nucleotide mutations, c.136A $>$ T, in exon 3, and c.1027T $>$ C, in exon 9. The nucleotide changes c.136A $>$ T and c. $1027 \mathrm{~T}>\mathrm{C}$ lead respectively to the ALDOB missense variants p.R46W and p.Y343H, are private mutations. The g.2840_9288del produces a null-allele (c.1_624del; p.M1?) which was identified in six unrelated HFI Italian patients.

DNA analysis in HFI studies is usually designed to look for the most frequent mutations in specific populations [Darsun et al., 2001; Kullberg-Lindh C et al., 2002; Esposito et al., 2004a; Kriegshäuser G et al, 2007; Davit-Spraul et al., 2008; Coffee et al., 2009]. However, by searching pertinent databases and publications, we found that 56 gene alterations, besides the novel ones described in this paper, thus far in individuals with HFI or with suspected HFI from diverse populations and ethnic backgrounds (Table 1). Therefore, sequencing of the whole $A L D O B$ gene is advisable to increase diagnostic sensitivity. Even in this latter case, one cannot completely rule out an 
$A L D O B$ alteration in HFI. This reasoning and our combined clinical and experimental observations prompted us to look for large deletions in the $A L D O B$ gene. To that aim, we constructed a three-step algorithm (Fig. 1) for the routine DNA analysis of HFI patients, which resulted in an analytical sensitivity of about $99 \%$ (Table 2) in our population. This strategy led to the identification of a number of subjects carrying an intragenic deletion, which was hitherto considered a very rare type of $A L D O B$ mutation - indeed, only two private, large $A L D O B$ gene deletions have been reported in HFI patients so far [Cross and Cox, 1990].

It is well known that small-segment PCR-based amplification methods mask deletions in heterozygotes. Indeed, according to both ARMS and sequence analysis, patient SR and her symptomatic brother appeared to be homozygous for the c.448G $>\mathrm{C}$ (p.A150P) mutation in exon 5, which is a common HFI genotype worldwide. Surprisingly, Mendelian transmission did not confirm heterozygosity in the father. We suspected that he could carry an intragenic deletion involving exon 5. To address this hypothesis, we analyzed $A L D O B$ gene regions by qPCR (Fig. 2A), in SR's father, and roughly mapped the proximal breakpoint to IVS1, and the distal breakpoint to IVS6. Using two primers positioned in IVS1 and IVS7, respectively, we obtained a $\sim 2.4-\mathrm{kb}$ amplification fragment containing the deletion junction. Sequencing showed that this deletion removed $6548 \mathrm{bp}$ (g.2840_9288del) that contain exons 2-6 (Fig. 2A, B). Given the fact that the ALDOB exon 2 contains the ATG initiation codon, g.2840_9288del should be a null-allele (c.1_624del; p.M1?). Specific amplification of the breakpoint junction revealed the deletion in both the symptomatic patients, who were therefore compound heterozygous for the c.448G $>C$ (p.A150P) mutation and the deletion (Fig. 3). We also found the deletion in one of the other two healthy siblings, who was an HFI carrier (Fig. 3), although he appeared normal by ARMS and sequencing.

Subject MG was the asymptomatic daughter of an HFI patient who was believed to be 
homozygous for the c.524C >A (p.A175D) mutation in exon 5, a common genotype in Italy [Esposito et al., 2004a]. Surprisingly, MG was normal by ARMS, in contrast with the a priori hypothesized condition of obligate carrier of the maternal mutation. We suspected that MG and her mother could carry an intragenic deletion involving exon 5 . Therefore, in both subjects, we looked for, and indeed found, the new g.2840_9288del deletion. MG did not show any other nucleotide substitution in either the whole $A L D O B$ coding region or the promoter, which we analyzed by sequencing. Interestingly, while preparing this paper, we identified four other patients who were compound heterozygotes for the new g.2840_9288del deletion and for the widespread HFI alleles, p.A150P or p.A175D, in exon 5. To our knowledge, this is the first report of six apparently unrelated patients sharing the same $A L D O B$ deletion. The g.2840_9288del deletion accounts for about $2.5 \%$ of all HFI alleles in our population (Table 2). It is reasonable to suspect that other cases of homozygosity for mutations located in exons 2-6 (e.g., c.370_373del, p.A150P, p.A175D, p.R204X) may be actually due to heterozygosity for a point mutation and a large deletion, the latter not being identifiable by conventional analytical methodology. Evaluation of Mendelian transmission in homozygous patients is necessary to avoid such erroneous results. Overall, our results indicate that, when carrying out the HFI molecular diagnostic protocol, investigators should be aware that the molecular epidemiology in their area may differ from that established due to the frequency of large intragenic deletions.

Large deletions are usually caused by unequal homologous recombination between short repetitive elements (SINE and LINE) or by non-homologous recombination involving DNA with minimal sequence homology [Antonorakis et al., 2000]. Alignment of the g.2840_9288del deletion junction with the $A L D O B$ germline sequence revealed highly repeated DNA sequences (SINEs) in introns 1 and 6 , and TTTAAA sequences in exon 6 and intron 6 . Furthermore, a palindromic sequence in IVS6, which could be involved in double-strand break events, is located just downstream the 
breakpoint junction (Fig. 2C). Therefore, non-homologous end-joining mechanisms may have played a role in this deletion [Toffolatti et al., 2002]. However, the fact that g.2840_9288del is a relatively common HFI allele, at least in Italy, suggests that it could be related to consanguineous marriages and genetic admixtures, with a founder effect in this geographic area, rather than to a recurrent mutation mechanism.

Following our algorithm (Fig. 1), when ARMS did not reveal any frequent point mutation in patients $\mathrm{AB}$ and $\mathrm{CL}$, we sequenced $A L D O B$ exons 2-9 including the adjacent splicing junctions and the promoter. We detected only one nucleotide substitution, in each patient. AB was heterozygous for c.1027T>C (p.Y343H) in exon 9 and CL for the nucleotide change c.136A>T (p.R46W) in exon 3 (reviewed but not shown). In both cases, Mendelian transmission confirmed that each proband inherited the mutation from one of the asymptomatic parents. We excluded that AB and CL could carry, on the other allele, the previously known $A L D O B$ partial deletions g.7516_9165del and g.9912_10836del [Cross and Cox, 1990]. In patient AB, we also excluded the presence of the new deletion g.2840_9288del described herein. The presence of both normal and mutated exon 3 in patient CL excluded the latter deletion a priori. It is noteworthy that the SNP database reports the c.136A > T (p.R46W) nucleotide variation as a rare polymorphism (dbSNP cluster ID \# rs41281039:A>T). However, we did not find either c.136A >T (p.R46W) or c.1027T>C (p.Y343H) in more than 300 alleles of subjects who did not appear to have HFI.

Twenty-six (44.8\%) of the 59 mutations identified in $A L D O B$ so far lead to amino acid substitutions. The new point mutations c.136A $>$ T (p.R46W) and c.1027T $>C$ (p.Y343H) involve residues, Arg46 and Tyr343, that are conserved in most vertebrate ALDOB sequences. Tyr343 is also conserved in the ALDOA and ALDOC sequences. In contrast, a previous study, based on an alignment of 21 known vertebrate aldolase sequences, identified ALDOB Arg46 as an isozyme- 
specific residue (ISR) [Pezza et al., 2003]. In fact, Arg46 is Ser in ALDOA and Gln in ALDOC. The conservation of ISRs among orthologs implies that they play a role in conferring the specific kinetic properties of each isozyme.

The effects of various HFI-related missense mutations on ALDOB function have been investigated by in vitro expression and functional characterization of the corresponding recombinant enzymes [Brooks and Tolan, 1994; Santamaria et al., 2000; Rellos et al., 2000; Esposito et al., 2002; Esposito et al., 2004a]. Thus, to assess whether the p.R46W and p.Y343H natural ALDOB variants are associated to a functional deficiency, we expressed the corresponding recombinant enzymes in bacterial cells. Both recombinant proteins were recovered in soluble form in bacteria grown at $37^{\circ} \mathrm{C}$, which indicates that substitutions do not produce major enzyme structural alterations. Therefore, we purified the His-tagged recombinant enzymes and then evaluated their kinetic properties (Table 3; Supp. Figure S1). All kinetic plots indicate Michaelian behavior. The $\mathrm{k}_{\mathrm{cat}}$ value of the p.R46W enzyme was $30 \%$ lower towards FBP and 70\% lower towards F1P compared to the wild type enzyme. Accordingly, the p.R46W specific activity ratio (FBP/F1P), which is 1.2 for normal ALDOB, was 2.8. The p.R46W $\mathrm{K}_{\mathrm{m}}$ for FBP was similar to that of the wild-type enzyme, but 4-fold higher than normal for F1P. Overall, the catalytic efficiency of p.R46W for F1P was more than 14fold lower than that of the wild type enzyme, whereas there was no relevant functional variation versus FBP. This is the first report of an ALDOB amino acid change that specifically affects F1P catalysis, without producing any functional alteration in FBP metabolism.

When assayed at $30^{\circ} \mathrm{C}$, the effects of the p.Y343H substitution on enzyme function are not as dramatic as those of p.R46W. Its $\mathrm{K}_{\mathrm{m}}$ and $\mathrm{k}_{\mathrm{cat}}$ values resembled that of the wild type enzyme for both substrates. Only the tiny increase in the mean specific activity ratio (FBP/F1P) would indicate that the p. $\mathrm{Y} 343 \mathrm{H}$ change slightly favors $\mathrm{FBP}$ catalysis at $30^{\circ} \mathrm{C}$. 
In addition, to evaluate metabolic effects of the novel ALDOB variants also in actual cells, we now start cloning ALDOB cDNA in a eukaryotic expression system.

Models of natural variants based on crystal structural data may also shed light on mutationinduced structural-functional alterations [Santamaria et al., 2000; Rellos et al., 2000; Esposito et al., 2002; Esposito et al., 2004a; Malay et al., 2005]. Analysis of the ALDOB three-dimensional structures clearly indicates that both Arg46 and Tyr343 are exposed to the solvent, far from the tetramer interface (Fig. 4A), and are consequently endowed with structural flexibility. The Arg46 side chain has very high B-factor values in all available ALDOB crystallographic models [Malay et al., 2005; Dalby et al., 2001]. Due to the external location, the replacement of Arg46 with a Trp does not produce any significant steric clash. A deeper analysis of the local context of Arg46 indicates that, although flexible, this residue is located in the same region as the Arg 304 side chain (Fig. 4B and C). Studies of ALDOA suggest that this residue may be involved in the binding of either the P-1 or the P-6 moiety of the substrates, presumably in different stages of the catalytic process [Blom and Sygusch, 1997; Choi et al., 1999; Choi et al., 2001; St-Jean et al., 2005; St-Jean et al., 2007; Sherawat et al., 2008]. From the structure of ALDOB, it is inferred that Arg 304 is involved in the binding of the P-1 moiety of FBP in intermediate states along the reaction (PDB 1FDJ, Fig. 4B). The positively charged Arg46 side chain generates electrostatic repulsion forces that may serve to confine Arg 304 in the correct position for substrate binding and catalysis (Fig. 4B). Therefore, it is not surprising that the replacement of Arg46 with an uncharged Trp residue has a remarkable impact on the enzyme activity toward F1P. On the other hand, the marginal alteration of the activity toward FBP may be ascribed to the presence of an additional anchoring group (the P-6 moiety) whose binding site is not altered by the mutation. To verify this interpretation, we reproduced and functionally analyzed two non-natural ALDOB recombinant variants of Arg46, namely p.R46K and 
p.R46A (Table 3), chosen with respect to the structural model. In p.R46K, the substitution of Arg46 with a positively charged Lys does not alter, even minimally, the enzyme activity towards F1P. In p.R46A, by changing Arg46 with an uncharged, albeit small Ala, we were able to reproduce perfectly the effect of the natural Arg46 to Trp substitution. These results strongly confirm that the loss of the positive charge of Arg is the main cause of F1P-specific malfunctioning observed in the p.R46W natural variant and not the steric bulk of Trp. Taken together, our functional and structural results confirm that Arg46 is an ALDOB ISR, since we have demonstrated that p.R46W is a variant enzyme with altered catalytic properties, towards F1P, the main form by which dietary fructose is transformed within the cell.

The limited functional effect of the p. $\mathrm{Y} 343 \mathrm{H}$ change recorded at $30^{\circ} \mathrm{C}$ is not surprising because the structural context of Tyr343 shows that this residue is located far from the active site, in a solvent-exposed pocket close to the enzyme surface, which precedes the highly flexible C-terminal region of ALDOB. In the crystal structure of the ALDOB ligand-free form, the Tyr343 side chain forms alternative interactions with the side chains Glu278 (Fig. 4D) or of His345 (Fig. 4E), in different subunits within the tetramer. The replacement of Tyr343 with a His residue (Fig. 4F) probably alters the local structure of the enzyme, but the flexibility of the surrounding region may attenuate the mutation-induced functional impact.

It is noteworthy that the C-terminal region of aldolases is crucial for substrate entry/release [Blom \& Sygusch, 1997; Esposito et al., 2004b]. In a previous study, we demonstrated that the ALDOA p.C338Y variant had functional alterations that were temperature-dependent [Esposito et al., 2005]. Cys338 resides in a switch region that precedes the mobile C-terminus in ALDOA, like Tyr343 in ALDOB. Therefore, we assayed the specific activity versus FBP and F1P of the wild-type, p.R46W and p.Y343H enzymes, at temperatures between $20^{\circ} \mathrm{C}$ and $48^{\circ} \mathrm{C}$. The activity of the p.Y343H mutant 
versus F1P was highest at $30^{\circ} \mathrm{C}$, whereas the activities of the wild type and p.R46W enzymes were highest at $42^{\circ} \mathrm{C}$ (Fig. $5 \mathrm{~A}$ and B). The p.Y343H enzyme was almost inactive at $45^{\circ} \mathrm{C}$ towards F1P, whereas the wild type enzyme retained high activity at this temperature. The reduction in specific activity associated to the temperature shift was less dramatic for FBP than for F1P (Fig. 5A).

To determine whether the functional observations were related to a temperature-dependent structure reorganization of ALDOB, we performed CD experiments on the recombinant wild type, p.R46W and p.Y343H enzymes (Fig. 5C and D). The three ALDOB forms display very similar CD spectra at $20^{\circ} \mathrm{C}$ (Fig. 5C). This result suggests that, in line with the functional and molecular graphics analysis, these mutations do not cause significant rearrangements of the overall structure of the enzyme. Thermal denaturation curves indicate that the three recombinant proteins have similar melting temperatures (Fig. 5D). However, the p.Y343H enzyme exhibited, as a distinctive feature, an evident variation of the $\mathrm{CD}$ signal at temperatures that precede the melting point. This suggests a local destabilization, and could be linked to the temperature-dependence of the specific activity of this variant enzyme. Overall, our results suggest that the Arg46Trp substitution does not affect enzyme stability, whereas a specific amino acid change (e.g. Tyr343His) in the ALDOB C-terminal domain may lead to temperature-dependent structural perturbations that alter F1P catalysis more severely than FBP catalysis. The latter finding is important in HFI-affected individuals. In fact, such variant enzymes may be temporarily inactivated during febrile episodes. The consequent alterations in fructose metabolism lead to the accumulation of fructose and F1P, which then exert their deleterious metabolic effects. In this context, it is noteworthy that most antipyretic agents given to children contain fructose or sucrose as sweetener.

Given the fact that HFI is not associated to specific phenotypic traits, we cannot confirm the clinical suspicion of HFI presence when only one well-known mutation is identified. This is 
especially true case when the mutation identified is a novel missense variant, as in the two cases reported here (patients $\mathrm{AB}$ and $\mathrm{CL}$ ). In vitro enzyme functional assays demonstrated that the two novel ALDOB variants, p.R46W and p.Y343H, had pathogenic potential; however, they were found in clinically symptomatic HFI subjects apparently carrying only one mutation. Interestingly, CattoSmith and Adams (1993) observed acute jaundice, gastrointestinal bleeding, hypoglycemia, proximal tubular acidosis, and disseminated intravascular coagulation in a patient who was inadvertently given fructose intravenously during the routine clinical-surgical management of other diseases. Liver biopsy of the patient showed that F1P aldolase activity was $30 \%$ of normal, and FBP aldolase activity was normal. In HFI patients, these values are between $0 \%$ and $6 \%$ and between $10 \%$ and $50 \%$ of normal, respectively. The authors hypothesized that the patient was an HFI heterozygous carrier that became ill only consequently to a massive fructose infusion. Over the past 20 years, we have analyzed numerous subjects that had a clinical diagnosis of HFI, and in a few cases we identified only one HFI-related genetic alteration. We would, therefore, suggest that acute symptoms of HFI can appear after fructose intake in usually asymptomatic heterozygotes. Therefore, care should be taken to avoid uncontrolled fructose administration and/or ingestion also in people who are heterozygous carriers of this recessive disease, as well as in individuals that are not yet known to be heterozygotes and have symptoms and/or signs of metabolic alterations that appear to be associated with chronic or unusually excessive fructose intake.

Currently, only molecular genetic techniques can identify heterozygous HFI subjects. The rational use of these techniques for screening programs requires prior knowledge of the frequencies of specific HFI alleles in the population under study. Therefore, given the deleterious long-term effects of exposure to fructose in low-fructose-metabolizer subjects, it is conceivable that, depending on the outcome of cost-benefit analyses, ALDOB gene sequencing could be conducted in diverse ethnic 
groups to understand better the molecular epidemiology of natural ALDOB gene variants. In turn, this might open the way to neonatal molecular screening programs [see also, James et al., 1996; Cox, 2002] as is the case of other nutritional diseases.

\begin{abstract}
Acknowledgements: Supported by a grant from Regione Campania (Convenzione CEINGERegione Campania, G.R. 27/12/2009), from Ministero dell'Istruzione, dell'Università e della Ricerca-Rome PS35-126/IND and from IRCCS - Fondazione SDN, and Ministero Salute, Rome, Italy. We are grateful to Jean Ann Gilder for revising and editing the text, and to Prof. Mario Masullo for advice about kinetic assays.
\end{abstract}

\title{
References
}

Ali M, Rosien U, Cox TM. 1993. DNA diagnosis of fatal fructose intolerance from archival tissue. Q J Med 86:25-30.

Ali M, Sebastio G, Cox TM. 1994a. Identification of a novel mutation (Leu 256-->Pro) in the human aldolase B gene associated with hereditary fructose intolerance. Hum Mol Genet 3:203-4.

Ali M, Tuncman G, Cross N, Vidailhet M, Bokesoy I, Gitzelmann R, Cox TM. 1994b. Null alleles of the aldolase B gene in patients with hereditary fructose intolerance. J Med Genet 31:499-503.

Ali M, Cox TM. 1995. Diverse mutations in the aldolase B gene that underlie the prevalence of hereditary fructose intolerance. Am J Hum Genet 56:1002-5.

Ali M, Rellos P, Cox T. 1998. Hereditary fructose intolerance. J Med Genet 35:353-365.

Antonorakis SE, Krawczak M, Cooper DN. 2000. The nature and mechanisms of human gene mutation. In: KW VBaK, editor. The genetic basis of human cancer. New York: McGraw Hill. 
Blom, N. and Sygusch, J. 1997. Product binding and role of the C-terminal region in class I Dfructose 1,6-bisphosphate aldolase. Nat. Struct. Biol. 4, 36-39.

Brooks CC, Buist N, Tuerck J, Tolan DR. 1991. Identification of a splice-site mutation in the aldolase B gene from an individual with hereditary fructose intolerance. Am J Hum Genet 49:1075-81.

Brooks CC, Tolan DR. 1994. A partially active mutant aldolase B from a patient with hereditary fructose intolerance. FASEB J 8:107-13.

Catto-Smith AG, Adams A. 1993. A possible case of transient hereditary fructose intolerance. J Inherit Metab Dis 16:73-77.

Choi KH, Mazurkie AS, Morris AJ, Utheza D, Tolan DR., Allen KA. 1999. Structure of a fructose1,6-bis(phosphate) aldolase liganded to its natural substrate in a cleavage-defective mutant at 2.3 Å. Biochemistry 38:12655-12664.

Choi KH, Shi J, Hopkins CE, Tolan DR, Allen KN. 2001. Snapshots of catalysis: the structure of fructose-1,6-(bis)phosphate aldolase covalently bound to the substrate dihydroxyacetone phosphate. Biochemistry 40:13868-13875.

Choi YK, Johlin FC, Summers RW, Jackson M, Rao SSC. 2003. Fructose Intolerance: An UnderRecognized Problem. Am J Gastroenterol 98:1348-1353.

Coffee EM, Yerkes L, Ewen EP, Zee T, Tolan DR. 2009. Increased prevalence of mutant null alleles that cause hereditary fructose intolerance in the American population. J Inherit Metab Dis 33:3342.

Cox TM. 2002. The genetic consequences of our sweet tooth. Nat Rev Genet 3:481-487.

Cross NC, Tolan DR, Cox TM. 1988. Catalytic deficiency of human aldolase B in hereditary fructose intolerance caused by a common missense mutation. Cell 53:881-5. 
Cross NC, Cox TM. 1990c. Partial aldolase B gene deletion in hereditary fructose intolerance. Am J Hum Genet. 47:101-106.

Cross NC, de Franchis R, Sebastio G, Dazzo C, Tolan DR, Gregori C, Odievre M, Vidailhet M, Romano V, Mascali G, et al.. 1990a. Molecular analysis of aldolase B genes in hereditary fructose intolerance. Lancet 335:306-9.

Cross NC, Stojanov LM, Cox TM. 1990b. A new aldolase B variant, N334K, is a common cause of hereditary fructose intolerance in Yugoslavia. Nucleic Acids Res 18:1925.

Dalby RD, Tolan DR, Littlechild JA. 2001. The structure of human liver fructose-1,6-bisphosphate aldolase. Acta Cryst D Biol Crystallogr 57:1526-1533.

Davit-Spraul A, Costa C, Zater M, Habes D, Berthelot J, Broué P, Feillet F, Bernard O, Labrune P, Baussan C. 2008. Hereditary fructose intolerance: frequency and spectrum mutations of the aldolase B gene in a large patients cohort from France--identification of eight new mutations. Mol Genet Metab 94:443-447.

Dazzo C, Tolan DR. 1990. Molecular evidence for compound heterozygosity in hereditary fructose intolerance. Am J Hum Genet 46:1194-9.

Dursun A, Kalkanoğlu HS, Coşkun T, Tokatli A, Bittner R, Koçak N, Yüce A, Ozalp I, Boehme HJ. 2001. Mutation analysis in Turkish patients with hereditary fructose intolerance. J Inherit Metab Dis 24:523-6.

Esposito G, Vitagliano L, Santamaria R, Viola A, Zagari A, Salvatore F. 2002. Structural and functional analysis of aldolase B mutants related to hereditary fructose intolerance. FEBS Lett 531:152-156.

Esposito G, Santamaria R, Vitagliano L, Ieno L, Viola A, Fiori L, Parenti G, Zancan L, Zagari A, Salvatore F. 2004a. Six novel alleles identified in italian hereditary fructose intolerance patients 
enlarge the mutation spectrum of the aldolase B gene. Hum Mutat 24:534.

Esposito G, Vitagliano L, Costanzo P, Borrelli L, Barone R, Pavone L, Izzo P, Zagari A, Salvatore F. 2004b. Human aldolase A natural mutants: relationship between flexibility of the C-terminal region and enzyme function. Biochem J 380:51-56.

Esposito G, Vitagliano L, Cevenini A, Amelio T, Zagari A, Salvatore F. 2005. Unraveling the structural and functional features of an aldolase A mutant involved in the hemolytic anemia and severe rhabdomyolysis reported in a child. Blood 105:905-6.

Gruchota J, Pronicka E, Korniszewski L, Stolarski B, Pollak A, Rogaszewska M, Ploski R. 2006. Aldolase B mutations and prevalence of hereditary fructose intolerance in a Polish population. Mol Genet Metab 87:376-8.

Hutchinson EG, Thornton JM. 1996. PROMOTIF -- a program to identify and analyze structural motifs in proteins. Protein Sci 5:212-220.

James CL, Rellos P, Ali M, Heeley AF, Cox TM. 1996. Neonatal screening for hereditary fructose intolerance: frequency of the most common mutant aldolase B allele (A149P) in the British population. J Med Genet 33:837-41.

Kajihara S, Mukai T, Arai Y, Owada M, Kitagawa T, Hori K. 1990 Hereditary fructose intolerance caused by a nonsense mutation of the aldolase B gene. Am J Hum Genet 47:562-7.

Kriegshäuser G, Halsall D, Rauscher B, Oberkanins C. 2007 Semi-automated, reverse-hybridization detection of multiple mutations causing hereditary fructose intolerance. Mol Cell Probes. 21:2268.

Kullberg-Lindh C, Hannoun C, Lindh M. 2002 Simple method for detection of mutations causing hereditary fructose intolerance. J Inherit Metab Dis. 25:571-5.

Laskowski RA, MacArthur MW, Moss DS, Thornton JM. 1993. PROCHECK: a program to check 
the stereochemical quality of protein structures. J Appl Cryst 26:283-291.

Malay AD, Allen KN, Tolan DR. 2005. Structure of the thermolabile mutant aldolase B, A149P: molecular basis of hereditary fructose intolerance. J Mol Biol 347:135-44.

Miller A, Adeli K. 2008. Dietary fructose and the metabolic syndrome. Curr Opin Gastroenterol 24:204-9.

Pezza JA, Choi KH, Berardini TZ, Beernink PT, Allen KN, Tolan DR. 2003. Spatial clustering of isozyme-specific residues reveals unlikely determinants of isozyme specificity in fructose-1,6bisphosphate aldolase. J Biol Chem 278:17907-17919.

Rellos P, Sygusch J, Cox TM. 2000. Expression, purification, and characterization of natural mutants of human aldolase B. Role of quaternary structure in catalysis. J Biol Chem 275:1145-51.

Salvatore F, Izzo P, Paolella G. 1986. Aldolase gene and protein families: structure, expression and pathophysiology. In: Blasi F, editor. Horizons in Biochemistry Biophysics, John Wiley and Sons. 8:611-665.

Sanchez-Gutierrez JC, Benlloch T, Leal MA, Samper B, Garcia-Ripoll I, Feliu JE. 2002. Molecular analysis of the aldolase B gene in patients with hereditary fructose intolerance from Spain. J Med Genet 39:e56

Santamaria R, Scarano M I, Esposito G, Chiandetti L, Izzo P, Salvatore F. 1993. The molecular basis of hereditary fructose intolerance in Italian children. Eur J Clin Chem Clin Biochem 31:675-678.

Santamaria R, Tamasi S, Del Piano G, Sebastio G, Andria G, Borrone C, Faldella G, Izzo P, Salvatore F. 1996. Molecular basis of hereditary fructose intolerance in Italy: identification of two novel mutations in the aldolase B gene. J Med Genet 33:786-788.

Santamaria R, Vitagliano L, Tamasi S, Izzo P, Zancan L, Zagari A, Salvatore F. 1999. Novel sixnucleotide deletion in the hepatic fructose-1,6-bisphosphate aldolase gene in a patient with 
hereditary fructose intolerance and enzyme structurefunction implications. Eur J Hum Genet 7:409-414.

Santamaria R, Esposito G, Vitagliano L, Race V, Paglionico I, Zancan L, Zagari A, Salvatore F. 2000. Functional and molecular modelling studies of two hereditary fructose intolerance-causing mutations at arginine 303 in human liver aldolase. Biochem J 350:823-828.

Santer R, Rischewski J, von Weihe M, Niederhaus M, Schneppenheim S, Baerlocher K, Kohlschütter A, Muntau A, Posselt HG, Steinmann B, Schneppenheim R. 2005. The spectrum of aldolase B (ALDOB) mutations and the prevalence of hereditary fructose intolerance in Central Europe. Hum Mutat 25:594.

Sherawat M, Tolan DR, Allen KN. 2008. Structure of a rabbit muscle fructose-1,6-bisphosphate aldolase A dimer variant. Acta Crystallogr D64:543-50.

Steinmann, B, Gitzelmann, R. 1981. The diagnosis of hereditary fructose intolerance. Helv Paediatr Acta 36:297.

Steinmann B, Gitzelmann R, Van den Berghe G. 2001. Disorders of fructose metabolism. In: Scriver CR, Beaudet al., Valle D, Sly WS, editors. The metabolic and molecular bases of inherited disease, 8th ed. New York:McGraw-Hill. p 1489-1520.

Stenson PD, Ball EV, Mort M, Phillips AD, Shiel JA, Thomas NS, Abeysinghe S, Krawczak M, Cooper DN. 2003. The Human Gene Mutation Database (HGMD): 2003 Update. Hum Mutat $21: 577-581$.

St-Jean M, Lafrance-Vanasse J, Liotard B, Sygusch J. 2005. High resolution reaction intermediates of rabbit muscle fructose-1,6-bisphosphate aldolase: substrate cleavage and induced fit. J. Biol. Chem. 280:27262-27270.

St-Jean M, Izard T, Sygusch J. 2007. Stereospecific proton transfer by a mobile catalyst in 
mammalian fructose-1,6-bisphosphate aldolase. J. Biol. Chem. 282:14309-14315.

Tolan DR. 1995. Molecular basis of hereditary fructose intolerance: mutations and polymorphisms in the human aldolase B gene. Hum Mutat 6:210-8.

Toffolatti L, Cardazzo B, Nobile C, Danieli GA, Gualandi F, Muntoni F, Abbs S, Zanetti P, Angelini C, Ferlini A, Fanin M, Patarnello T. 2002. Investigating the mechanism of chromosomal deletion: characterization of 39 deletion breakpoints in introns 47 and 48 of the human dystrophin gene. Genomics 80:523-30.

Van Der Spoel D, Lindahl E, Hess B, Groenhof G, Mark AE, Berendsen HJ. 2005. GROMACS: fast, flexible, and free. J Comput Chem 26:1701-18. 


\section{Figure 1}

HFI molecular diagnosis algorithm. The first step (by ARMS methodology) looks for the mutations c.448G>C (p.A150P), c.524C>A (p.A175D), c.356_359delCAAA (p.N120K121del), c.672T>A (p.Y204X), c770T>C (p.L257P), c.1005C>G (p.N335K), c.1013C>T (p.A338V). The second step (whole coding region sequencing) analyzes patients who have only one or no mutation detected by ARMS. The third step applies to: (a) patients who showed only one mutation; and (b) patients who result, by PCR standard procedures $\left(1^{\text {st }}\right.$ and $2^{\text {nd }}$ steps $)$, homozygous for mutations located in exons 26, and for whom Mendelian transmission data were either unavailable or not consistent with autosomal recessive inheritance. In the latter case, the third step should improve molecular analysis in subjects for which routine test already confirmed the clinical suspicion.

\section{Figure 2}

A. ALDOB gene structure and size of the novel g.2840_9288del (c.1_624del) variant. Horizontal arrows indicate the approximate position of the primers (9380 bp apart, in the normal allele) used in the long-PCR that resulted in the isolation of an amplification fragment spanning the deletion junction (2932 bp long, 6548 bp shorter than normal) in the mutated allele (HFI_del). IVS1pBP and IVS6dBP indicate the position of the proximal and distal breakpoints of the deletion along the gene. B. Sequence electropherogram of the deletion junction site (arrow). C. Alignment of the DNA sequence spanning the deletion junction (BRPJ) with the corresponding normal $A L D O B$ intron regions shows that the 5' breakpoint resides in IVS1 and the 3' breakpoint in IVS6 (numbering according to the GenBank \# NT_008470.13 sequence). The arrow indicates the breakpoint site. In yellow, palindromic sequence; green and cyan: repeated sequences. 


\section{Figure 3}

Pedigree of the SR's family in which the g.2840_9288del (c.1_624del) deletion was detected, and results of the duplex amplification used to distinguish between carriers of the deletion and normal individuals (357 bp: internal amplification control; 258 bp: deletion-specific amplicon; see Material and Method). Symbols' patterns indicate the four different genotypes, in the family members.

\section{Figure 4}

Structural features of normal and variant human ALDOB. A. Location of Arg46 (blue circles) and Tyr343 (pink circles) in the three-dimensional structure of the ALDOB tetramer. The four subunits are differently colored. B. Structural context of positively charged Arg46. Although this residue is quite flexible, it influences the position of nearby positively charged Arg304, which is involved in the binding of the P-1 moiety of substrates. C. Model of mutant p.R46W. Bulkier Trp, which can be easily accommodated without steric overlaps, provokes an alteration compatible with a difficulty in the normal substrate binding. D. Structural context of Tyr343, which is located in a pocket close to the enzyme surface. Its side chain forms a strong interaction with Glu278 in one subunit. E. Structural context of Tyr343 within another subunit (red colored) of the tetramer. Its side chain forms a strong interaction with the His345 side chain. F. Model of mutant p.Y343H.

\section{Figure 5}

Specific activities and circular dichroism analysis of ALBOB variants as function of temperature. A. Specific activities of p.Y343H (v), and B. p.R46W $(\sigma)$ compared to the wild-type $(\lambda)$ enzyme versus FBP (solid lines) and F1P (dotted lines), measured at increasing temperature (between $20^{\circ} \mathrm{C}$ and $\left.50^{\circ} \mathrm{C}\right)$. Standard deviation derives from variability associated with three repeats of the experiment. C. 
CD spectra for Y343H (v), p.R46W ( $\sigma)$ compared to the wild type $(\lambda)$. Experiments were carried out in $20 \mathrm{mM}$ TrisHCl (pH 7.5) and 1.5 mM DTT. D. Denaturation curves plotted by transforming the $\mathrm{CD}$ signal at $222 \mathrm{~nm}$ in the fraction folded (fraction folded $=[\theta]_{222}(\mathrm{~T}) /[\theta]_{222}\left(20{ }^{\circ} \mathrm{C}\right)$ ).

\section{Figure S1}

Selected double-reciprocal plots for recombinant natural enzymes. Data from: p.Y343H (v), p.R46W $(\sigma)$, wild-type $(\lambda)$ recombinant enzyme. Assays performed with FBP (solid lines) and F1P (dotted lines). 
Table 1: HFI-related mutations in $A L D O B$

\begin{tabular}{|c|c|c|c|c|}
\hline & Nucleotide change & Exon & Deduced effect & Refrences \\
\hline 1 & c. $2 \mathrm{~T}>\mathrm{C}$ & 2 & p.M1T & Ali et al.., 1993 \\
\hline 2 & c. $10 \mathrm{C}>\mathrm{T}$ & 2 & p.R4X & Ali et al.., 1994b \\
\hline 3 & c.62delA & 2 & p.Q21RfsX36 & Santamaria et al.., 1996 \\
\hline 4 & c. $113-1 \mathrm{G}>\mathrm{A}$ & IVS2 & deduced splicing defect & Santer et al.., 2005 \\
\hline 5 & c.113-1Gdel113_115delGGTA & IVS2/exon 3 & deduced splicing defect & Steinmann et al.., 2001 \\
\hline 6 & c.1_624del (found in patients SR and MG) & $2-6$ & p.M1? & this work \\
\hline 7 & c.136A $>$ T (found in patient $\mathrm{CL}$ ) & 3 & p.R46W & this work \\
\hline 8 & c.146delT & 3 & p.V49GfsX27 & Davit-Spraul A et al.., 2008 \\
\hline 9 & c. $170 \mathrm{G}>\mathrm{C}$ & 3 & p.R57P & Davit-Spraul A et al.., 2008 \\
\hline 10 & c. $178 \mathrm{C}>\mathrm{T}$ & 3 & p.R60X & Brooks and Tolan, 1994 \\
\hline 11 & c. $250 \mathrm{delC}$ & 3 & p.L84SfsX24 & Gruchota et al.., 2006 \\
\hline 12 & c. $221 \mathrm{~T}>\mathrm{C}$ & 3 & p.I74T & Esposito et al.., 2004a \\
\hline 13 & c. $324 \mathrm{G}>\mathrm{A}$ & 3 & p.K108K (deduced splicing defect) & Sánchez-Gutiérrez et al.., 2002 \\
\hline 14 & c. $325-1 \mathrm{G}>\mathrm{C}$ & IVS4 & deduced splicing defect & Esposito et al.., 2004a \\
\hline 15 & g.7516-9165del & $4-5$ & p.L109_S160del & Cross \& Cox, 1990 \\
\hline 17 & c. $331 \mathrm{C}>\mathrm{T}$ & 4 & p.Q111X & Esposito et al.., 2004a \\
\hline 18 & c. $345 \_372$ del 28 & 4 & p.L116FfsX26 & Santer et al.., 2005 \\
\hline 19 & c.360_363delCAAA & 4 & p.N120KfsX30 & Dazzo et al.., 1990 \\
\hline 20 & c. $400 \mathrm{C}>\mathrm{A}$ & 5 & p.R134S & Santer et al.., 2005 \\
\hline 21 & c. $403 \mathrm{~T}>\mathrm{C}$ & 5 & p.C135R & Brooks and Tolan, 1994 \\
\hline 22 & c. $442 \mathrm{~T}>\mathrm{C}$ & 5 & p.W148R & Ali \& Cox, 1995 \\
\hline 23 & c. $444 \mathrm{G}>\mathrm{A}$ & 5 & p.W148X & Davit-Spraul A et al.., 2008 \\
\hline 24 & c. $448 \mathrm{G}>\mathrm{C}$ & 5 & p.A150P & Cross et al.., 1988 \\
\hline 25 & c. $488 \mathrm{C}>\mathrm{T}$ & 5 & p.A163V & Santer R et al.., 2005 \\
\hline 26 & c. $497 \mathrm{~A}>\mathrm{T}$ & 5 & p.E166V & Steinmann et al.., 2001 \\
\hline 27 & c. $522 \mathrm{C}>\mathrm{G}$ & 5 & p.Y174X & Gruchota et al.., 2006 \\
\hline 28 & c. $523 \mathrm{G}>\mathrm{A}$ & 5 & p.A175T & Cross et al.., 1990a \\
\hline 29 & c. $524 \mathrm{C}>\mathrm{A}$ & 5 & p.A175D & Steinmann et al.., 2001 \\
\hline 30 & c. $532 \mathrm{~T}>\mathrm{C}$ & 5 & p.C178R & Santer R et al.., 2005 \\
\hline 33 & g.9912_10836del & $6-7$ & p.N181_G267del & Steinmann et al.., 2001 \\
\hline 34 & c.548_552del & 6 & p.L183_V184del & Santamaria R et al.., 1999 \\
\hline 35 & c. $554 \mathrm{C}>\mathrm{G}$ & 6 & p.P185R & Sánchez-Gutiérrez et al.., 2002 \\
\hline 36 & c. $612 \mathrm{~T}>\mathrm{A}$ & 6 & p.Y204X & Ali et al.., 1993 \\
\hline 37 & c. $612 \mathrm{~T}>\mathrm{G}$ & 6 & p.Y204X & Santer et al.., 2005 \\
\hline 38 & c. $625-1 \mathrm{G}>\mathrm{A}$ & IVS6 & deduced splicing defect & Ali et al.., 1994b \\
\hline 39 & c. $625-2 \mathrm{~A}>\mathrm{G}$ & IVS6 & deduced splicing defect & Esposito et al.., 2004a \\
\hline 40 & c. $665 \mathrm{G}>\mathrm{T}$ & 7 & p.V222F & Esposito et al.., 2004a \\
\hline 41 & c. $686 \mathrm{~T}>\mathrm{C}$ & 7 & p.L229P & Esposito et al.., 2004a \\
\hline 42 & c.689insTGCTAA & 7 & p.L229_K230insNA & Davit-Spraul A et al.., 2008 \\
\hline 43 & c. $720 \mathrm{C}>\mathrm{A}$ & 7 & p.C240X & Kajihara et al.., 1990 \\
\hline 44 & c. $770 \mathrm{~T}>\mathrm{C}$ & 7 & p.L257P & Ali et al.., 1994b \\
\hline 45 & c. $799+2 \mathrm{~T}>\mathrm{A}$ & IVS7 & deduced splicing defect & Santer R et al.., 2005 \\
\hline 46 & c. $839 \mathrm{C}>\mathrm{A}$ & 8 & p.A280P & Davit-Spraul A et al.., 2008 \\
\hline 47 & c.841_842delAC & 8 & p.S281TfsX52 & Santer et al.., 2005 \\
\hline 48 & c. $851 \mathrm{~T}>\mathrm{C}$ & 8 & p.L284P & Santer et al.., 2005 \\
\hline 49 & c. $865 \mathrm{delC}$ & 8 & p.L289FfsX8 & Cross et al.., 1990a \\
\hline 50 & c. $911 \mathrm{G}>\mathrm{A}$ & 8 & p.R304Q & Santamaria R et al.., 2000 \\
\hline 51 & c. $910 \mathrm{C}>\mathrm{T}$ & 8 & p.R304W & Tolan, 1995 \\
\hline 52 & c. $932 \mathrm{~T}>\mathrm{C}$ & 8 & p.L311P & Davit-Spraul A et al.., 2008 \\
\hline 53 & c.953_994del42bp & 8 & p.A318_A332del & Davit-Spraul A et al.., 2008 \\
\hline 54 & c.1000_1005delGCTAAC & 9 & p.A335_N336del & Steinmann et al.., 2001 \\
\hline 55 & c.[1000_1006delGCTAACT; 1008_1009insTG] & 9 & p.N335AfsX29 & Brooks et al.., 1991 \\
\hline 56 & c. $1005 \mathrm{C}>\mathrm{G}$ & 9 & p.N335K & Cross et al.. 1990b \\
\hline 57 & c. $1013 \mathrm{C}>\mathrm{T}$ & 9 & p.A338V & Ali et al.., 1998 \\
\hline 58 & c.1027T $>C$ (found in patient $A B)$ & 9 & p.Y343H & this work \\
\hline 59 & c.[1038_1039insACACT; 1044_1049delTTCTGG] & 9 & p.G347TfsX27 & Santer et al.., 2005 \\
\hline
\end{tabular}


Table 2: HFI-Italian alleles and their frequencies*

\begin{tabular}{|c|c|c|}
\hline Nucleotide change & Deduced effect & Frequency $(\%)$ \\
\hline c. $448 \mathrm{G}>\mathrm{C}$ & p.A150P & 37.30 \\
\hline c. $523 \mathrm{G}>\mathrm{A}$ & p.A175T & 32.79 \\
\hline c. $612 \mathrm{~T}>\mathrm{A}$ & p.Y204X & 7.79 \\
\hline c.360_363delCAAA & p.N120KfsX30 & 4.51 \\
\hline c. $1005 \mathrm{C}>\mathrm{G}$ & p.N335K & 3.69 \\
\hline c.1_624del & p.M1? (deduced absent protein) & 2.46 \\
\hline c.770T $>\mathrm{C}$ & p.L257P & 2.05 \\
\hline c. $625-2 \mathrm{~A}>\mathrm{G}$ & deduced splicing defect & 0.82 \\
\hline c. $1013 \mathrm{C}>\mathrm{T}$ & p.A338V & 0.82 \\
\hline c. 62 delA & p.Q21RfsX36 & 0.41 \\
\hline c.136A $>\mathrm{T}$ & p.R46W & 0.41 \\
\hline c. $221 \mathrm{~T}>\mathrm{C}$ & p.I74T & 0.41 \\
\hline c. $325-1 \mathrm{G}>\mathrm{C}$ & deduced splicing defect & 0.41 \\
\hline c.314_315insGGGGATCGTGGT & p.K97_G98insGIVV & 0.41 \\
\hline c. $331 \mathrm{C}>\mathrm{T}$ & p.Q111X & 0.41 \\
\hline c. $345 \_372$ del 28 & p.L116FfsX26 & 0.41 \\
\hline c. $497 \mathrm{~A}>\mathrm{T}$ & p.E166V & 0.41 \\
\hline c.548_552del & p.L183_V184del & 0.41 \\
\hline c. $665 \mathrm{G}>\mathrm{T}$ & p.V222F & 0.41 \\
\hline c. $686 \mathrm{~T}>\mathrm{C}$ & p.L229P & 0.41 \\
\hline c. $865 \mathrm{delC}$ & p.L289FfsX8 & 0.41 \\
\hline c. $911 \mathrm{G}>\mathrm{A}$ & p.R304Q & 0.41 \\
\hline c. $910 \mathrm{C}>\mathrm{T}$ & p.R304W & 0.41 \\
\hline c.1030T $>C$ & p.Y343H & 0.41 \\
\hline Total analytic sensitivity** & & 98,77 \\
\hline
\end{tabular}


Table 3 Kinetic parameters of the recombinant human ALDOB enzymes analyzed

\begin{tabular}{lccccccccc}
\hline Enzyme & \multicolumn{2}{c}{$K_{\mathrm{m}}$} & \multicolumn{2}{c}{$\begin{array}{c}k_{\text {cat }} \\
\left(\mathrm{s}^{-1}\right)\end{array}$} & $\begin{array}{c}\text { FBP/F1P } \\
\text { ratio }\end{array}$ & \multicolumn{2}{c}{$\begin{array}{c}\text { Catalytic efficiency (CE) } \\
k_{\text {cat }} / K_{\mathrm{m}}\end{array}$} & \multicolumn{2}{c}{ CE fold change } \\
& FBP & F1P & FBP & F1P & & FBP & F1P & FBP & F1P \\
\hline Wild type & $0.95 \pm 0.13$ & $0.73 \pm 0.25$ & $0.84 \pm 0.08$ & $0.71 \pm 0.08$ & 1.2 & $0.89 \pm 0.04$ & $1.03 \pm 0.26$ & \\
p.R46W & $0.76 \pm 0.13$ & $3.07 \pm 0.80$ & $0.58 \pm 0.05$ & $0.21 \pm 0.05$ & 2.8 & $0.77 \pm 0.07$ & $0.07 \pm 0.002$ & 1.1 & $14.2^{2}$ \\
p.Y343H & $1.12 \pm 0.23$ & $1.01 \pm 0.25$ & $1.10 \pm 0.05$ & $0.73 \pm 0.08$ & 1.5 & $1.02 \pm 0.17$ & $0.81 \pm 0.04$ & 0.9 & 1.2 \\
p.R46K & $0.92 \pm 0.27$ & $1.22 \pm 0.18$ & $1.08 \pm 0.07$ & $0.89 \pm 0.10$ & 1.2 & $1.23 \pm 0.30$ & $0.73 \pm 0.03$ & 0.8 & 1.3 \\
p.R46A & $0.81 \pm 0.18$ & $2.73 \pm 0.34$ & $0.71 \pm 0.11$ & $0.19 \pm 0.07$ & 3.7 & $0.89 \pm 0.06$ & $0.07 \pm 0.17$ & 1.0 & $13.9^{2}$
\end{tabular}

Assays were performed at $30^{\circ} \mathrm{C}$. Kinetic measures were repeated three (non-natural variants) or six times (natural enzymes) and $\mathrm{K}_{\mathrm{m}}$ and $\mathrm{k}_{\text {cat }}$ values were calculated from double-reciprocal plots with the use of a least-squares method. FBP/F1P ratio indicates the ratio between the mean specific activity obtained for each of the two substrates. Standard deviation derives from variability obtained after multiple repeats of the experiment. ${ }^{1}$ nonnatural variant. ${ }^{2} \mathrm{P}$-value $<0.01$ 


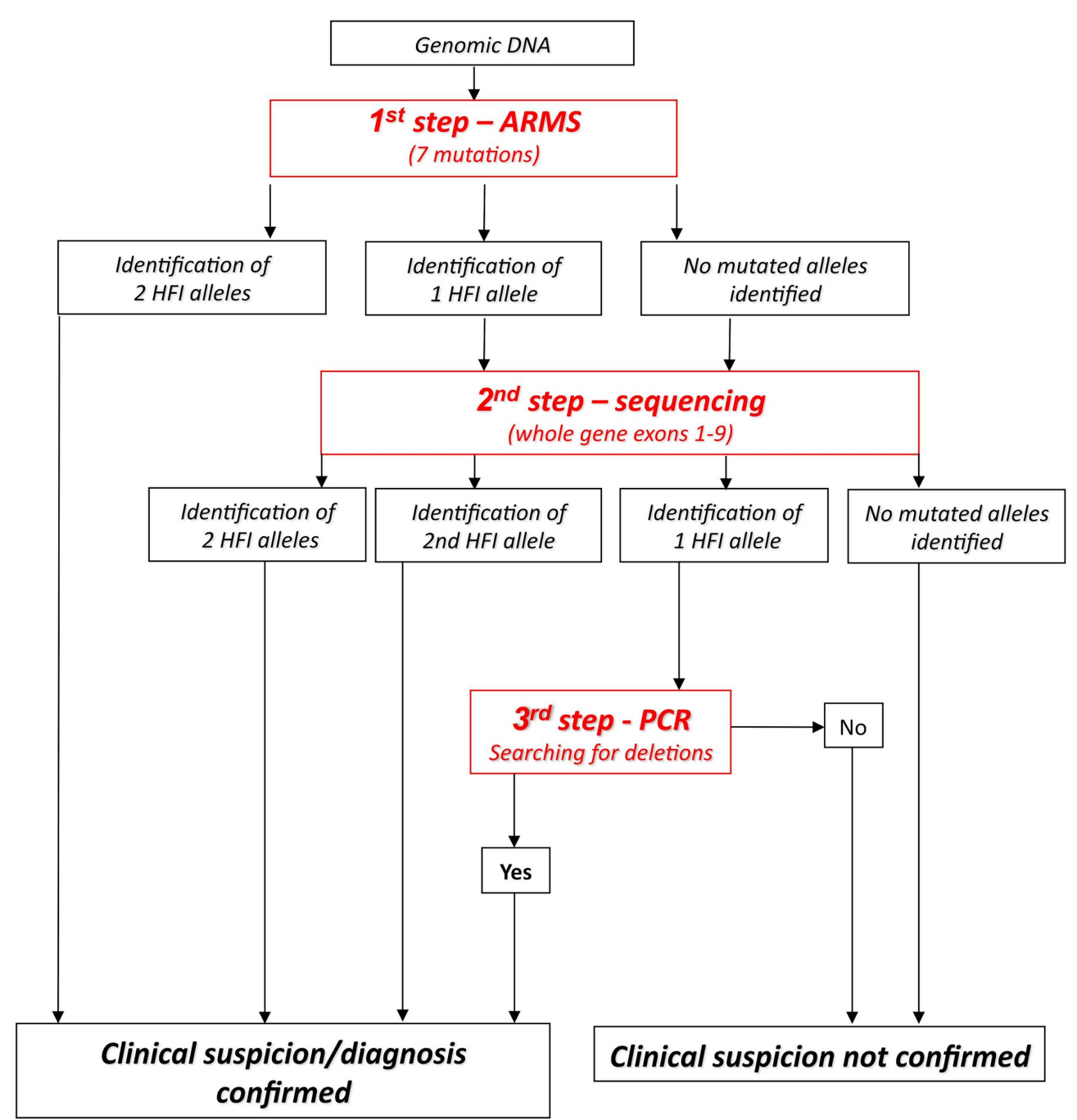

$237 \times 250 \mathrm{~mm}(300 \times 300 \mathrm{DPI})$

John Wiley \& Sons, Inc. 


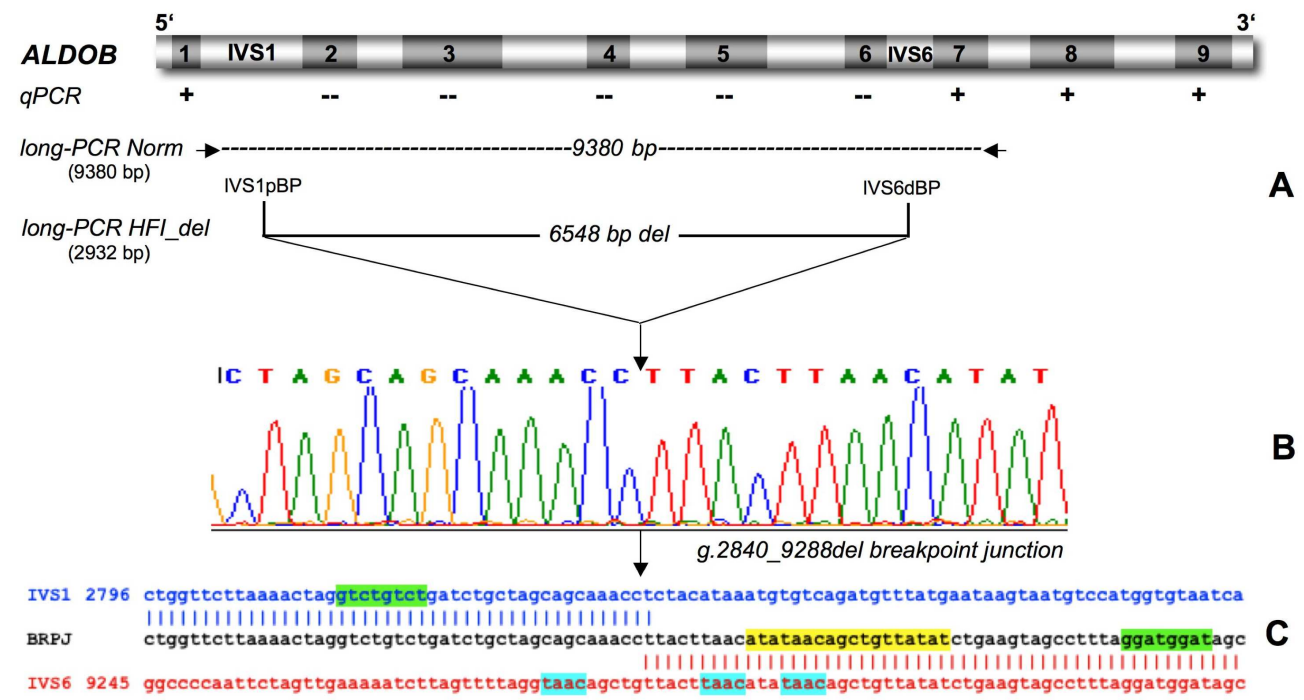

$249 \times 138 \mathrm{~mm}(300 \times 300$ DPI $)$

John Wiley \& Sons, Inc. 


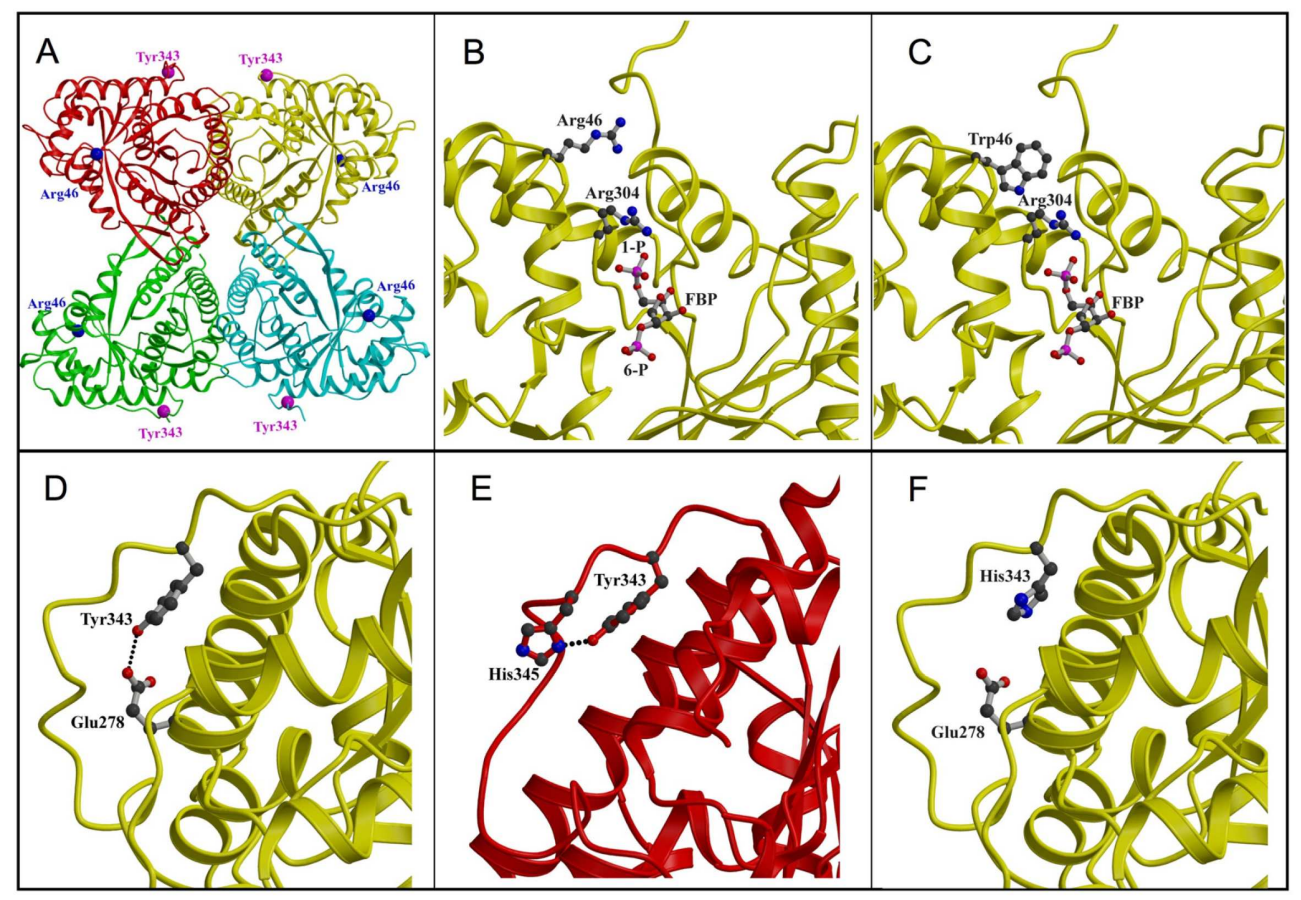

$199 \times 139 m m(300 \times 300$ DPI $)$

John Wiley \& Sons, Inc. 


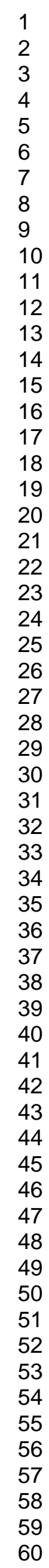
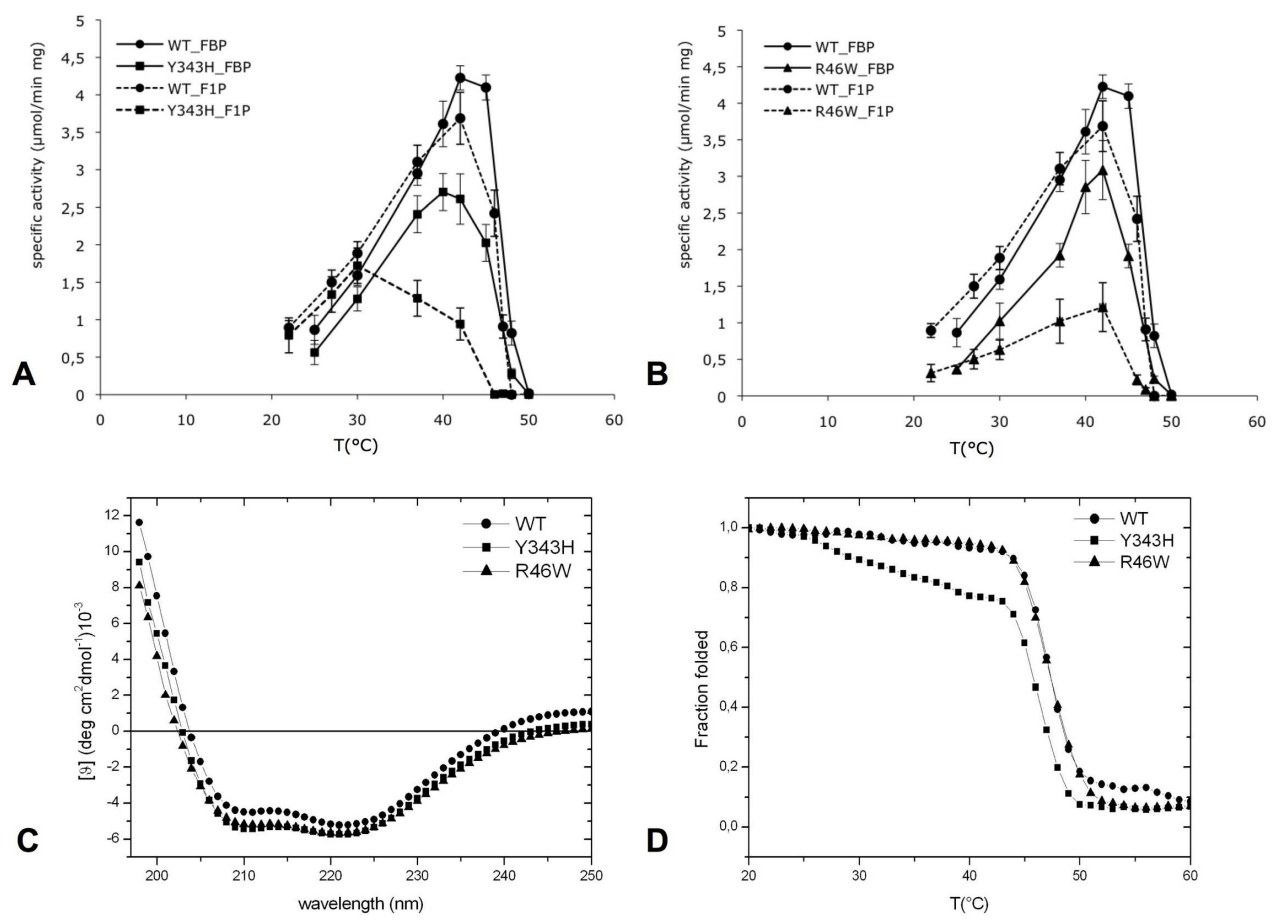

$199 \times 149 \mathrm{~mm}(300 \times 300 \mathrm{DPI})$

John Wiley \& Sons, Inc. 

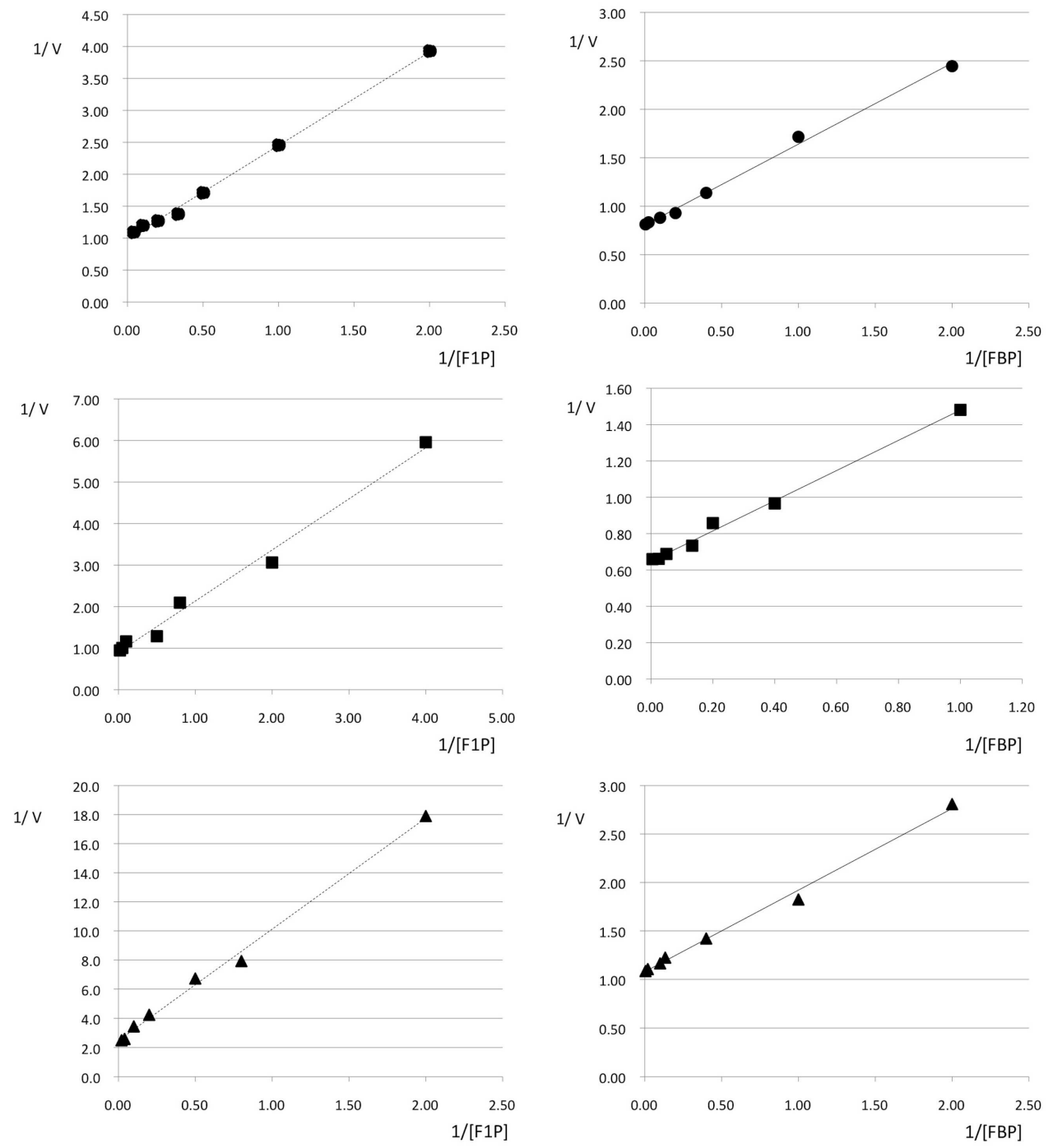

$231 \times 250 \mathrm{~mm}(300 \times 300$ DPI $)$

John Wiley \& Sons, Inc. 